\title{
Towards deeper comprehension in higher engineering education: "method of cornerstones"
}

\author{
Aki Korpela ${ }^{1}$, Timo Tarhasaari², Lauri Kettunen², Risto Mikkonen² and Hanna Kinnari- \\ Korpela ${ }^{1}$ \\ ${ }^{1}$ Department of Electrical Engineering, Tampere University of Applied Sciences, Tampere, Finland \\ 2 Electromagnetics, Tampere University of Technology, Tampere, Finland \\ For correspondence: aki.korpela@tamk.fi
}

\begin{abstract}
During the current millennium, universities have faced a new kind of problem: there is not enough higher learning in higher education. Driving forces have mainly been economical, since financial pressure and effort for increasing efficiency have given rise to growing amount of accessed and graduated students. In addition, a pressure for accelerated graduation has driven universities to decrease the emphasis on thorough learning of fundamentals. Consequently, during recent decades so called immediate skills have gained excessive role in comparison with longterm skills in engineering education. This is problematic, since the roots of higher learning in engineering are closely related to the sovereign understanding of fundamentals. In our previous paper, we suggested a method to promote higher learning in engineering education, and now we concretise those ideas through a simple case study of electrical engineering. In our example we demonstrate how the cornerstones of modelling are utilised to clarify and to deepen the learning of simplest circuit analysis cases of electrical engineering. However, since the models of natural sciences tend to have a hierarchical structure, method of cornerstones can be applied to many fields of engineering. Recently, a study carried out at MIT ended up to call for "coherent and interconnected curriculum structure" to achieve excellence in engineering education. We suggest that by utilising the hierarchical structure of natural sciences in engineering education, such a cohenernt and interconnected structure can be created. In this paper, we present how method of cornerstones can be used to build such a structure.
\end{abstract}

Keywords: higher engineering education, higher learning, cornerstones of modelling, electrical engineering

\section{Introduction}

Recently, a global worry about the lack of higher learning in higher education has arisen (Keeling 2011, Sullivan 2008, Sheppard 2008). In engineering, the concept of higher learning is closely related to understanding how abstractions are exploited to tackle tangible problems, and consequently, "learning to think" (Bok 2006, Shulman 1972). In our previous paper, we proposed a method to promote higher learning by means of cornerstones of modelling (Korpela et al. 2015). In this paper, we concretise those ideas via a simple case study of electrical engineering.

During the current millennium, political decisions steering the universities have generally included the following characteristics (Mortenson 2012):

1. Public funding has decreased. Universities and their research units have to be more efficient.

2. The amount of accessed students in universities has increased. At the same time, a growing share of accessed students should graduate in accelerated schedule.

3. According to a recent trend, engineering degrees have to be more wide-ranging than before.

All the listed items easily steer universities towards the tendency, where specialised studies are seen more important than the thorough and time-consuming learning of fundamentals. If the importance 
of fundamentals gets underrated, instead of deeper comprehension, learning in specialised studies may easily produce only immediate skills without an important aspect of thorough understanding. The problem with immediate skills is in their potential expiration: their validity may expire over the technological development. As "learning to think" is closely related to the eagerness of lifelong learning, we should not underrate the importance of fundamentals in engineering education. Without thorough understanding, lifelong learning will only half measure, since the generalisation of immediate skills to tackle everyday and often interdisciplinary problems is burdensome. In the end, there are no shortcuts to higher learning. In engineering, only the thorough understanding of fundamentals creates a sustainable foundation for deeper comprehension.

In the study carried out at Massachusetts Institute of Technology, Dr Ruth Graham ended up to conclude that achieving excellence in engineering education calls for "coherent and interconnected curriculum structure" (Graham 2012). In engineering, the hierarchical structure of natural sciences enables a coherent way to organise the substance. The fundamental idea is illustrated in figure 1, which presents the development of different fields of engineering from the theory of everything. Although this coherent model of intensive research still remains incomplete, it inevitably seems that all the fields of engineering fundamentally arise from the same foundation (Anderson 1972).

As we approach the theory of everything from any field of engineering, generality increases. As a consequence, different fields of engineering resemble each other more and more. For example, in electrical engineering the level of electromagnetic fields and waves is more general than the level of circuit analysis. No matter how well you master circuit analysis, it doesn't really give you the ability to understand other fields of engineering. However, if you master electromagnetic fields and waves, you will already have quite strong premises to understand also other fields of engineering, for example hydraulics. The dashed lines in figure 1 represent different levels of modelling, or more precisely, different levels of concretisation. When we recede from theory of everything, level of concretisation increases, since more and more details get fixed. And at the same time, generality decreases. Each level of concretisation includes a set of fundamental rules of modelling, which we call cornerstones. We suggest that by identifying the cornerstones of modelling on each level of concretisation, and by finding out the relations between the cornerstones of different levels, a coherent and interconnected curriculum structure can be created.

For example, in the simplest resistive circuit analysis of electrical engineering, the cornerstones are Kirchhoff's laws and Ohm's law. And as a hierarchical step towards the theory of everything is taken, Maxwell's equations and constitutive laws lay the foundation for electromagnetic modelling. Thus, they are the new cornerstones on the next level of lower concretisation. And most importantly, the

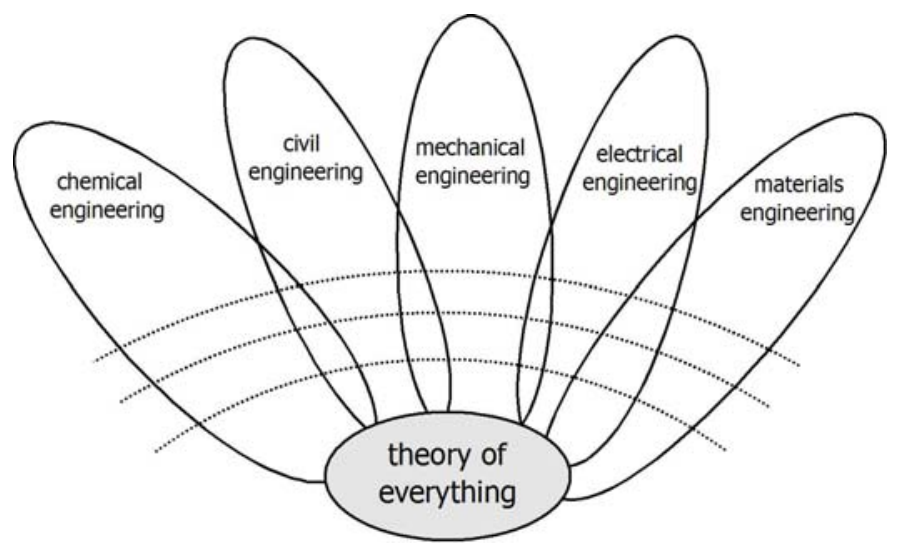

Figure 1. Some fields of engineering and their development from the theory of everything. Dashed lines represent the levels of concretisation. 
cornerstones on the higher level of concretisation (Kirchhoff's law and Ohm's law) only arise from the ones of more general level (Maxwell's equations and constitutive laws). Since this kind of hierarchical structure is inherent in natural sciences, the concept of cornerstones enables a coherent way to organise the substance in many fields of engineering education.

The balance between immediate and long-term skills is an endless dilemma in engineering education. In order to offer students important engineering skills appreciated in various tasks of industry, the toolbox of an engineer is filled with different designing tools, rules, standards and measurement procedures. These constitute immediate skills in engineering education. They are certainly important, but they also have a downside: their validity may expire over the technological development. In order to also gain more profound understanding and readiness for the changes in rapidly evolving industry, careful and time-consuming learning of fundamentals is required. These constitute longterm engineering skills, or even permanent engineering skills, since their feasibility is not affected by the changes of technology. Over the time, the development of immediate skills in engineering education has quite successfully followed the changes in technology. However, we suggest that this is not the case with long-term engineering skills. Especially in the economic pressure of current millennium, engineering education has excessively tilted towards immediate skills. Hence, we suggest that changes in engineering curriculum have not promoted a sustainable development of long-term skills during the most recent decades. In fact, the topical concept of wide-ranging engineering degrees is fundamentally closely related to long-term skills and higher learning. As the very purpose of science is to understand and to explain the world with as small amount of principles as ever possible, the concept of wide-ranging arises from the ability to understand and to exploit abstractions.

The lack of higher learning has been widely recognised, but according to our observations, a systematic method to promote higher learning in engineering education does not exist. Educational research in engineering has mainly focused on the important development of learning environments, learning methods and pedagogical skills (Brown 2005, Jonassen 2012, Kinnari-Korpela 2015, Porter 2014). In our previous paper, we presented the ideas of the method to promote higher learning in higher engineering education by means of cornerstones (Korpela et al. 2015). In this paper, we bring those ideas into more concrete level through a simple example of electrical engineering. In addition, we demonstrate how cornerstones can be used to build a coherent and interconnected curriculum structure in engineering education.

\section{Traditional way of circuit analysis education}

Now, we slightly aggravate by calling the following way of circuit analysis education as a "traditional

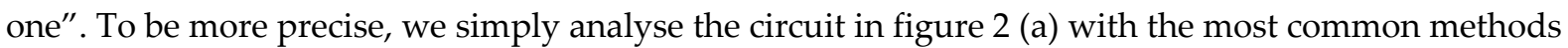
of circuit analysis without really paying attention to their foundations. Here, we use the methods of mesh currents, node voltages and Thevenin equivalent. The assignment is to determine the current in the middle branch of the circuit. In order to concretise the exercise, let's give the following values for the components: $R_{1}=10 \Omega, R_{2}=20 \Omega, R_{12}=15 \Omega, E_{1}=20 \mathrm{~V}, E_{2}=25 \mathrm{~V}$.

\section{Mesh-current method}

When the circuit in figure 2 (a) is analysed with the mesh-current method, mesh currents are set in each essential mesh of the circuit. Essential mesh is a loop that does not contain any other loop. Because the circuit in figure 2 (a) has two essential meshes, we need two mesh currents, the direction of which can be arbitrarily chosen. If the mesh currents $I_{a}$ and $I_{b}$ are set as in figure $2(b)$, the meshcurrent equations can be written as 


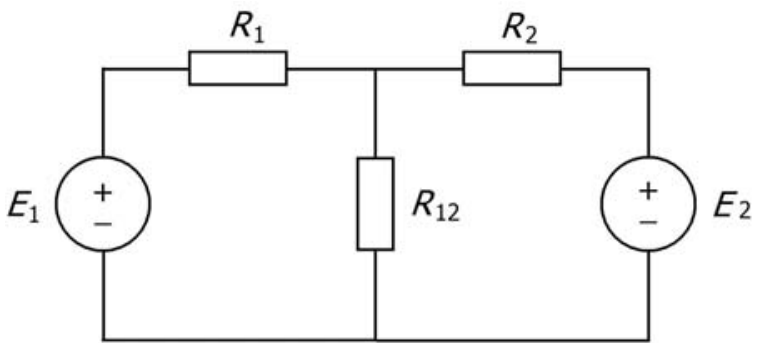

(a)

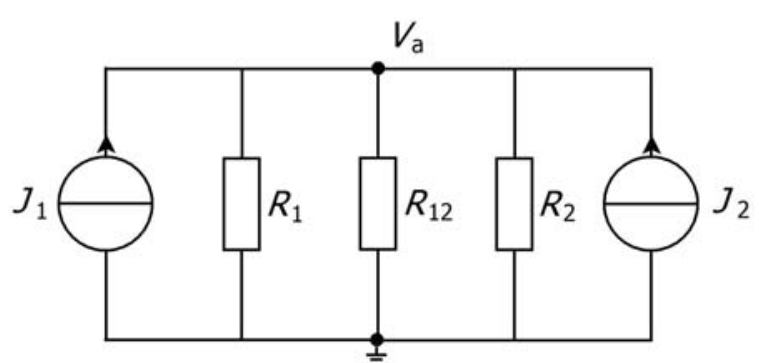

(c)

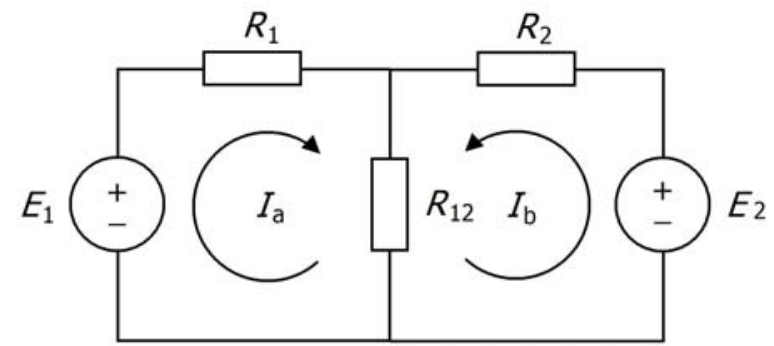

(b)

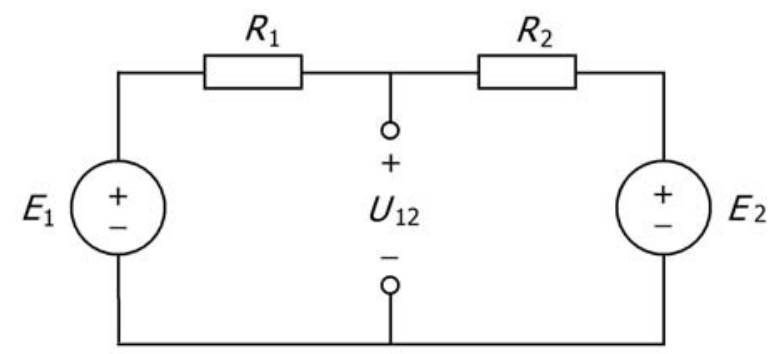

(d)

Figure 2. (a) Simple circuit model modified for (b) mesh-current method, (c) node-voltage method, and (d) Thevenin equivalent.

$$
\left\{\begin{array}{c}
\left(R_{1}+R_{12}\right) I_{\mathrm{a}}+R_{12} I_{\mathrm{b}}=E_{1} \\
R_{12} I_{\mathrm{a}}+\left(R_{2}+R_{12}\right) I_{\mathrm{b}}=E_{2}
\end{array} .\right.
$$

With the chosen mesh currents, the current in the middle branch of the circuit, $I \mathrm{~m}$, can be written as

$$
I_{\mathrm{m}}=I_{\mathrm{a}}+I_{\mathrm{b}} .
$$

By substituting the component values, we get $I_{\mathrm{m}}=1 \mathrm{~A}$ in the downward direction of figure 2 (a).

\section{Node-voltage method}

When the circuit in figure 2 (a) is analysed with the node-voltage method, the first task is to find the number of unknown potentials. From figure 2 (a) we can see that the number of different potentials is four, but two of these aren't actually unknown since the source voltages are known. The situation is more illustrative in figure 2 (c), where the voltage sources have been transformed into the current sources. Now, it is easy to identify that the circuit has two unknown electric potentials, thus it has two essential nodes. In the node-voltage method, one essential node is chosen as the ground reference. In figure 2 (c), the bottom node has been grounded. Then, unknown potentials are assigned for the remaining essential nodes. After choosing the ground reference, the circuit in figure 2 (c) has only one unknown potential, $V$ a. Now, the node-voltage equation can be written as

$$
\left(\frac{1}{R_{1}}+\frac{1}{R_{12}}+\frac{1}{R_{2}}\right) V_{\mathrm{a}}=J_{1}+J_{2} .
$$

With the chosen values of the components, from equation (3) we get $V_{a}=15 \mathrm{~V}$. Now, the downward current of the resistor $R_{12}$ can be written as

$$
I_{\mathrm{m}}=\frac{V_{\mathrm{a}}-0}{R_{12}} .
$$


As a result, we get the same 1 A current as in mesh-current method.

Thevenin equivalent

When the circuit in figure 2 (a) is analysed with the Thevenin equivalent, the first step is to identify its terminals. Since the target is to find the current in the middle branch of the circuit, the terminals arise from disconnecting the middle branch. Thus, as presented in figure 2 (d), they are the nodes existing over the open circuit of the resistor $R_{12}$. In the method of Thevenin equivalent, the circuit in figure 2 (d) is presented as an equivalent circuit, or a black box, which consist only of one voltage source and one resistor connected in series. The terminals have to be fixed, since the equivalence of different circuits can only be determined from fixed nodes. In general, Thevenin equivalent, or black-boxing, is an important tool in electrical engineering, since we are typically only interested in the external behaviour of electric circuits.

Now, we can find the Thevenin equivalent for the circuit in figure 2 (d) by merging the components. First, we transform the voltage sources into current sources, as presented in figure 3 (a). Observed from the nodes, parallel-connected resistors and current sources can now be merged, as presented in figure 3 (b). Then, another source transformation results in Thevenin equivalent, figure 3 (c). As the assignment was to determine the current in the middle branch of the original circuit, the resistor $R_{12}$ is returned between the nodes in figure $3(\mathrm{~d})$. Now, the current $I_{\mathrm{m}}$ can be simply determined from

$$
I_{\mathrm{m}}=\frac{E_{\mathrm{Th}}}{R_{\mathrm{Th}}+R_{12}} .
$$

As a result, we get the same 1 A current as in mesh-current and node-voltage methods.

When students solve the problem of figure 2 (a) with the methods of mesh-currents, node-voltages and Thevenin equivalent, it is probable that their immediate engineering skills get developed.

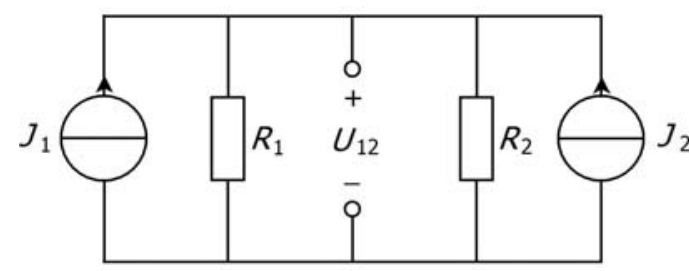

(a)

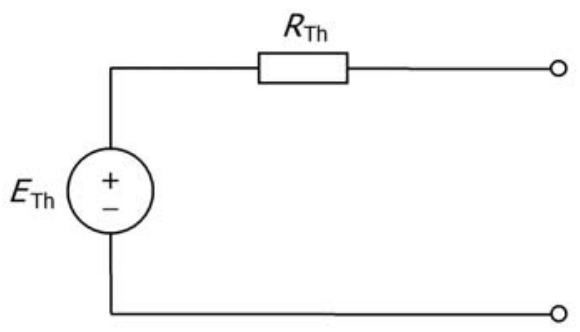

(c)

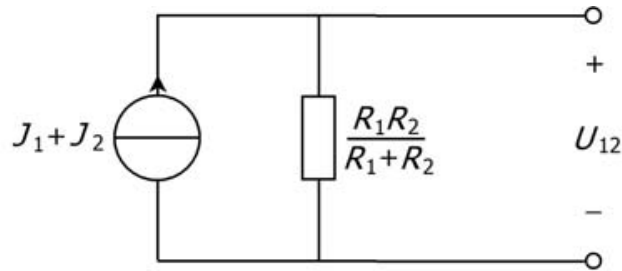

(b)

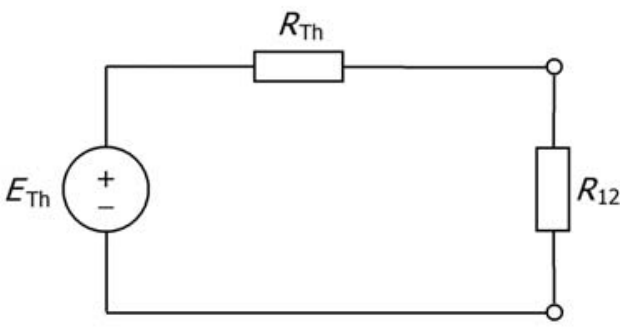

(d)

Figure 3. Progress of Thevenin equivalent to determine the current of $R_{12}$ in figure 1 (a): (a) transformation of voltage sources into current sources, (b) merging the components,

(c) transformation of current source into voltage source, and (d) return of $R_{12}$.

However, this aggravated example of education doesn't provide any means to understand the assumptions validating the methods of modelling. In that sense, the development of long-term engineering skills remains insufficient. 


\section{"Method of cornerstones" in circuit analysis education}

Identification and implementation of cornerstones

Now, we'll include the idea of cornerstones in the resistive circuit analysis education, which was earlier called as a "traditional one". The implementation of the method starts with the identification of cornerstones. In this example, we are dealing with resistive DC circuits. As a consequence, we have only three cornerstones: Kirchhoff's voltage law, Kirchhoff's current law, and Thevenin-Nortonequivalents for the components.

At first, we determine the current $I_{\mathrm{m}}$ by considering the cornerstones as unchallenged rules. According to Kirchhoff's voltage law, the sum of voltages across a closed loop is zero. And according to Kirchhoff's current law, the sum of currents at any node is zero. With Thevenin-Norton equivalents we mean the current-voltage relations for all the elements of the circuit. In the circuit of figure 2 (a) the elements are voltage sources, resistors, conductors, and also air. Voltage sources are assumed to be ideal, hence their voltages are constant regardless of current. The current-voltage relation for resistors is Ohm's law, and conductors are assumed to be ideal. Thus, they conserve the potential without any losses. Also the element of air has to be taken into account, since we assume that no current flows in the air regardless of voltage.

When the cornerstones of modelling have been identified, we possess all the necessary rules to analyse any resistive DC circuit. Thus, any circuit can be analysed by means of cornerstones - no additional methods are required. This is one important observation that students of electrical engineering should learn: the justification of different methods in circuit analysis only arises from possible reduction of workload.

Now, let's analyse the circuit in figure 2 (a) by using the cornerstones. In order to use Kirchhoff's laws, we first need to identify the branch currents and fix their orientations. As can be seen from figure 2 (a), we have three branch currents, the orientation of which can be arbitrarily chosen. We'll use here the orientation presented in figure 4 , and since we have three branch currents, we need three equations to solve the problem. Since the number of essential meshes is two in the circuit of figure 4, we can write two linearly independent equations of Kirchhoff's voltage law. And the remaining equation comes from Kirchhoff's current law. Thus, we get

$$
\left\{\begin{array}{c}
U_{\mathrm{R} 1}+U_{\mathrm{R} 12}-E_{1}=0 \\
U_{\mathrm{R} 2}+U_{\mathrm{R} 12}-E_{2}=0 . \\
I_{1}+I_{2}=I_{\mathrm{m}}
\end{array} .\right.
$$

And after applying Ohm's law for the resistor voltages

$$
U_{\mathrm{R} 1}=R_{1} I_{1}, U_{\mathrm{R} 12}=R_{12} I_{\mathrm{m}}, U_{\mathrm{R} 2}=R_{2} I_{2},
$$

we get the same $I_{\mathrm{m}}=1$ A as previously. 




Figure 4. Thevenin-Norton equivalents for the components and choice for the orientation of branch currents.

Figure 4 also presents Thevenin-Norton equivalents for the components. The elements of conductor and air are included in five components of the circuit, hence we have five Thevenin-Norton equivalents with two nodes in each. Due to Thevenin-Norton equivalents, we know the currentvoltage relations between all the nodes in figure 4. And after applying Kirchhoff's laws, any circuit can be analysed. Consequently, in the end, circuit analysis is nothing but Kirchhoff's laws and Thevenin-Norton equivalents (Baez 2015).

\section{Clarifying the education by means of cornerstones}

In order to clarify "the traditional way of circuit analysis education" from students' point of view, the role of cornerstones has to be made apparent in each method of modelling. Thus, next we'll show that different methods of modelling are not independent rules, but instead, they only arise from different applications of cornerstones. In the mesh-current method, the equations of Kirchhoff's voltage law are written with mesh currents instead of branch currents. Consequently, the number of unknown variables decreases, and the mesh currents can be solved by using Kirchhoff's voltage law only. However, Kirchhoff's current law is utilised when the branch currents are solved from the mesh currents. Thus, the mesh-current method splits the problem into two parts. First the mesh currents are solved with Kirchhoff's voltage law, after which the branch currents are solved with Kirchhoff's current law.

In the node-voltage method, the equations of Kirchhoff's current law are written with node potentials. In general, the number of linearly independent equations of Kirchhoff's current law in any pathwise connected circuit is $n-1$, where $n$ is the number of essential nodes. And by choosing the ground reference for one essential node, the number of unknown potentials in any circuit reduces to $n-1$. Consequently, branch voltages can now be solved by using the equations of Kirchhoff's current law only. Thus, all the equations of node-voltage method arise from Kirchhoff's current law. And from figure 2 (c) we can easily see that Kirchhoff's voltage law for any essential mesh results in

$$
\left(V_{\mathrm{a}}-0\right)+\left(0-V_{\mathrm{a}}\right)=0 .
$$

Hence, in the node-voltage method the branch voltages are solved by using the equations of Kirchhoff's current law. Furthermore, any closed loop in the circuit automatically also fulfils Kirchhoff's voltage law.

In the method of Thevenin equivalent, the idea is to replace the original circuit with the simplest equivalent one. The word equivalent refers to the characteristics of the circuit measured from the chosen terminals. For example, the circuits in figures 2 (d) and 3 (c) operate equivalently when measured from the chosen terminals. Required steps from the original circuit to Thevenin equivalent usually include operations such as merging the components and transforming the sources. However, 
the rules for these operations come from Kirchhoff's laws only. Thus, the method of Thevenin equivalent also arises from the applications of Kirchhoff's laws.

Pursuing deeper comprehension by looking underneath the cornerstones

Despite the field of engineering, the competence of fluent and flawless modelling is an important part of engineering skills. However, in order to gain deeper comprehension and "learn to think" (Bok 2006), we suggest that attention should also be paid to the assumptions behind the validity of cornerstones. In this way, we also promote the development of long-term engineering skills.

When we model resistive DC circuits, we typically stand on the cornerstones. That is, we treat the cornerstones as unchallenged rules whose validity is not questioned. However, in order to promote long-term engineering skills, we suggest that engineering education should include at least a peek towards the next level of lower concretisation. (Wieman 2013) In our case study of resistive DC circuits, a hierarchical step towards increasing generality is presented in figure 5.

The aim of looking underneath the cornerstones is to see them as testable properties instead of unchallenged rules. That is, we try to give students an impression about the assumptions validating the cornerstones. However, when students are studying the course of resistive DC circuits, they typically have no clue about electromagnetic fields or Maxwell's equations. Hence, this is where the role of a teacher becomes especially important. From students' point of view, a teacher has to be able to use a familiar language while introducing the topics on the lower level of concretisation. Typically a mathematical representation is not a familiar one. Thus, in order to verbalise the cornerstones on the lower level of concretisation, deeper content knowledge of a teacher is called for. Next, we'll give an example of verbalising the assumptions behind the cornerstones of resistive DC circuits.

According to Kirchhoff's voltage law, the voltage of any closed loop is zero. This rule arises from Faraday's law, which can be verbalised in the following way: "The vortex of electric field is associated with time-varying magnetic flux density." As a vortex depicts a region in which a flow is mostly rotating around an axis, the voltage of a closed loop will not be zero, if the vortex of electric field exists. Hence, the validity of Kirchhoff's voltage law requires a constant magnetic flux density. In the analysis of resistive DC circuits, we are dealing with constant currents and voltages. Thus, nothing varies with time. However, the validity of Kirchhoff's voltage law cannot be determined from the



Figure 5. Cornerstones of modelling in the levels of resistive DC circuits and electromagnetic fields and waves.

properties of the circuit only. Instead, external conditions have to be included also. For example, if a resistive DC circuit is located near a transformer, changing magnetic flux density deletes the validity 
of Kirchhoff's voltage law. In addition, an interesting detail is that when investigating for example AC circuits, Kirchhoff's voltage law is not exactly valid anymore, although it is commonly utilised.

According to Kirchhoff's current law, the sum of currents flowing into a node is equal to the sum of currents flowing out from the same node. This rule arises from Ampères law, which can be verbalised in the following way: "Current density and time-varying electric flux density are associated with the vortex of magnetic field." In order to understand Kirchhoff's current law, we first assume that the effect of time-varying electric flux density is negligible. Then, Ampères law can be verbalised as: "Current density is associated with the vortex of magnetic field." In addition, the validity of Kirchhoff's current law presumes the following conditions: 1) electric charge is conserved, 2) current flows only in conductors, and 3) the density of electric charge does not change. The first condition can be assumed generally valid, but the validity of the others is not self-evident at all. For example, the changes in charge density result in the violation of Kirchhoff's current law, since the current is determined as passing charge per unit time. However, in circuit analysis we usually neglect this detail by applying Kirchhoff's current law to a single point with no physical volume. Then, we also neglect the changes in charge density. However, as in Kirchhoff's voltage law, the validity of Kirchhoff's current law depends also on the surroundings of the circuit. For example, by applying a varying electric flux density to a resistive DC circuit, Kirchhoff's current law is violated.

As an example of verbalising Thevenin-Norton equivalents, let's take a closer look at Ohm's law of circuit analysis. It arises from the corresponding constitutive law combining the electric field and current density.

- Voltage between the nodes $a$ and $b$ results from the line integral of electric field from $a$ to $b$. If the electric field is assumed to be constant, the voltage will simply be the product of electric field and the distance between $a$ and $b$.

- Current results from the surface integral of current density. If the current density is assumed to be constant in the cross-section of conductor, the current will simply be the product of current density and cross-sectional area.

- Resistance of an object depends on the material property called resistivity and the dimensions of an object. When resistivity is multiplied by object's length and divided by its cross-sectional area, we get the value for object's resistance.

When we model the operation of a resistor with Ohm's law only, it is also worth noting that at the same time we neglect inductive and capacitive phenomena. As in Kirchhoff's laws, the validity of these assumptions is not only an inner property of the circuit, but it also depends on the surroundings of the circuit.

This was an example of looking underneath the cornerstones. By understanding their premises, students are given better prerequisites to gain deeper understanding and as a result, to achieve higher learning and long-term engineering skills. Consequently, as the cornerstones are seen as testable properties instead of unchallenged rules, a typical phrase "in theory yes but not in practice" becomes mostly irrelevant (Korpela et al. 2015).

More generally, the substance in engineering education is mostly about identifying the concretisations of modelling. Regardless of field of engineering, it is truly important to recognise the concretisations that education in different situations relies on. Then we can have the cornerstones, and then we can build a coherent structure for engineering education, as Graham (2012) called for.

\section{Benefits of method of cornerstones}

In this paper, we gave an example about the utilization of cornerstones in the development of engineering education. The goal was 1) to clarify the content, and 2) to deepen the comprehension. 
The aggravated example presented here was dealing with electrical engineering, but the method can be generalised in many branches of engineering. For example, in the basic courses of classical mechanics Newton's laws are the cornerstones of modelling. And when we take a look at the next level of lower concretisation, we are dealing with the laws of conservation of momentum, energy and angular momentum.

After the implementation of resistive circuit analysis courses in technical university, a common feedback from students has dealt with the confusion related to a great amount of different methods of modelling. According to students, benefits of different methods easily remain unclear, and it is often difficult to see, which method of modelling is the most suitable for a certain circuit. However, by utilising the idea of cornerstones in the education of resistive circuit analysis, the problem of multiple methods of modelling dissipates, since all the different methods arise from the same cornerstones. Thus, all the methods of modelling can just be seen as different ways to apply the cornerstones. In this way, students can understand that the justification of existence of different methods comes from finding the most efficient way (the smallest number of equations) to solve the problem. This is the idea behind the clarification of education by means of method of cornerstones.

Regardless of the field of engineering, method of cornerstones also offers a systematic method to promote higher learning. When cornerstones have been identified and utilised in modelling, we highly suggest that at least a peek underneath the cornerstones is also taken. Then, instead of unchallenged rules, cornerstones can be seen as testable properties. At the same time, due to increasing awareness of the assumptions validating the cornerstones, the content knowledge gets deeper. In the end, we are worried about increasing superficiality in engineering education. Instead of superficiality and immediate skills, we suggest to invest in more profound approach in order to also gain deeper comprehension and long-term engineering skills. Thus, as the fundamental purpose of science is to see more from less, we want to include its undeniable benefits also in engineering education (Anderson 1972). In general, we propose that engineering education in a certain level of concretisation should always also contain a view towards the next level of lower concretisation.

The idea of coherent and interconnected curriculum structure is presented in figure 6. Three levels of concretisation are presented, and for example, they could represent the levels of modelling in the curriculum of undergraduate engineers. The scientific foundation of degree is built on the lowest level of concretisation, and then the main methods of modelling in the field are studied on the next level of higher concretisation. Finally, the highest level of concretisation represents the models of specialised studies. As an example, the bachelor degree of electric power engineering follows quite well the structure of figure 6. The scientific foundation is built from Maxwell's equations (lowest level), and the majority of modelling in the field takes place on the level of circuit analysis (middle level). Finally, specialised studies of electric power engineers include some more complicated models of power grids and electric machines (highest level).

Coherence and interconnection of curriculum structure can be achieved by identifying the cornerstones of modelling on each level of concretisation, and furthermore, by understanding the relations between the cornerstones of different levels. In the best case, students can understand how a single cornerstone on the lowest level of concretisation is related to a single method of modelling in specialized studies. These details are highlighted with dark grey filling in figure 6. More generally, if the substance of engineering curricula can be presented with the principles presented in figure 6 , a great step towards coherent and interconnected structure will be taken. 


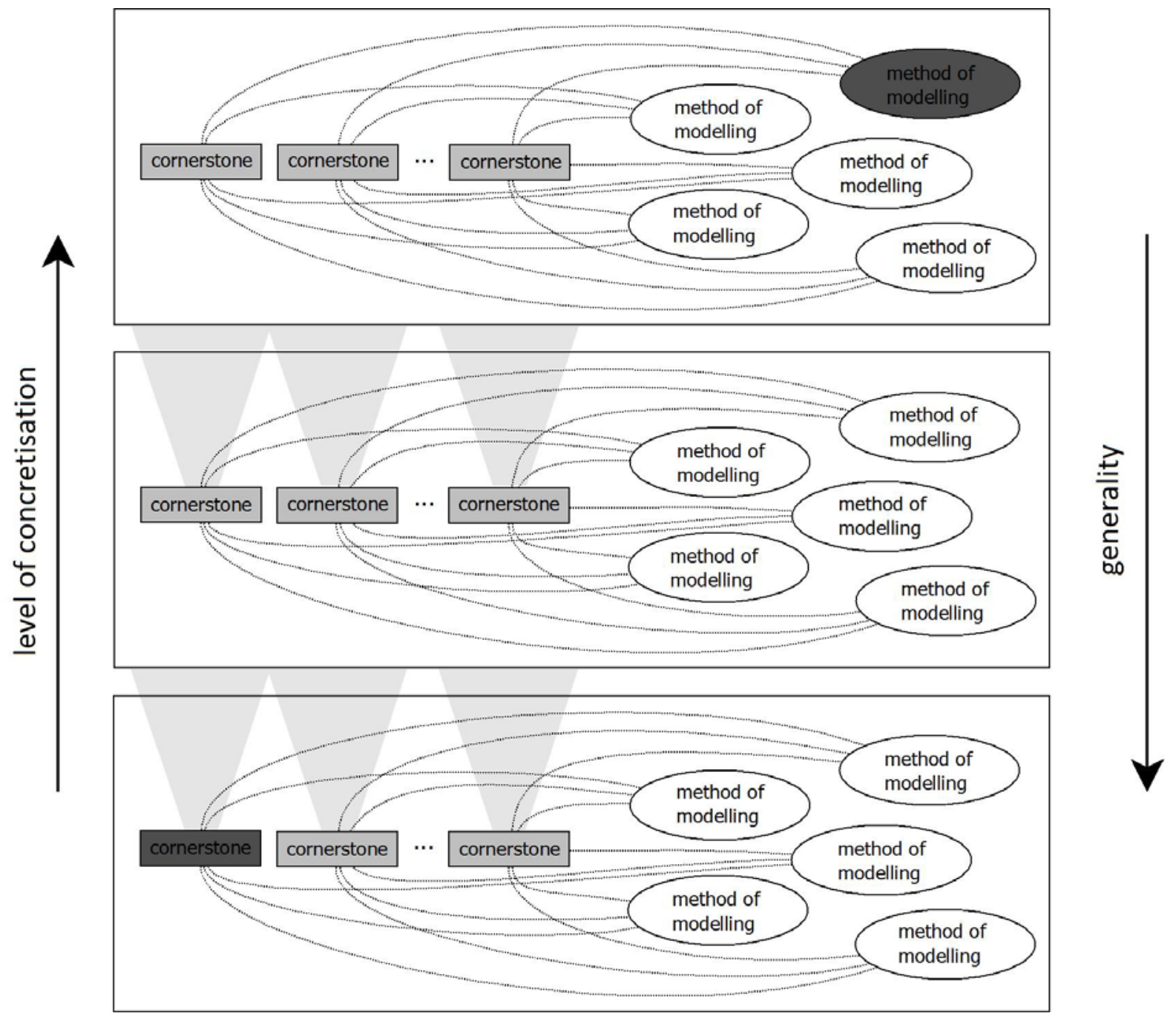

Figure 6. The idea of coherent and interconnected curriculum structure in engineering education.

\section{Discussion}

The dilemma between immediate and long-term engineering skills is not a new one. Since the emphasis of engineering is on getting devices and systems to run, it is important that engineering degrees include a fair amount of immediate tools. On the other hand, it is also clear that competitive $R \& D$ requires a more profound approach by the means of deeper comprehension. In addition, since validity of some immediate engineering skills may expire over the technological development, longterm skills offer readiness for changes in rapidly evolving industry. According to our view, in the economic pressure of current millennium immediate skills have gained excessive emphasis in engineering education. The trend has also been driven by society's belief that by investing in quantity of accelerated graduation we'll get increasing know-how for industry. However, we suggest that there are no shortcuts in achieving deeper comprehension, which really creates the only sustainable soil for increasing engineering know-how. Instead of engineers with immediate skills only, competitive industry requires deeper comprehension and the skills of logical reasoning. As a combination, we really get engineering graduates that, with the description of Derek Bok, have "learned to think" (Bok 2006).

According to our experience, some fields of engineering have already drifted quite far away from their roots. To be precise, in some specific and narrow fields of engineering, even professionals do not necessarily know where their models originate from. Thus, they are not aware about the 
concretisation of the models they are using. As a consequence, harmful shortcuts in education may come out. Although these are only cautionary examples, yet they make us worried about such progress becoming more common. Luckily, these problems are avoided by recognising the concretisations and identifying the cornerstones of modelling.

Another ongoing trend in engineering education is wide-ranging degrees. The original idea is reasonable, since the goal is to prepare students to better encounter the increasing interdisciplinary of engineering industry. However, the success of implementation is questionable, since it tends to gain ground from the thorough learning of fundamentals. At least in Finland, a wide-ranging degree of engineering has been interpreted to include studies from growing variety of fields. With this progress, the relative share of introductory studies tends to increase. As the very purpose of science is to understand and to explain the world with as small amount of principles as ever possible, the concept of wide-ranging fundamentally arises from the ability to understand and to exploit abstractions. Thus, we suggest that by investing in deeper comprehension, also the general insight of science gets wider. As the level of concretisation gets lower, and as generality increases, the fundamental purpose of science, to see more from less, gets more and more significant role also in education.

\section{Conclusions}

In the economic pressure of current millennium, universities have generally invested in the quantity of accelerated graduation. At the same time, a global worry about the lack of higher learning in higher engineering education has arisen. The problem is closely related to the balance between immediate and long-term skills in the degrees of engineering. Since the investments in quantity and rate of graduation tend to underrate a thorough learning of fundamentals, the balance has recently shaken excessively towards immediate engineering skills. In our opinion, this does not result in increasing know-how for industry. Instead, more emphasis in engineering education should be put on deeper comprehension and long-term engineering skills. The lack of higher learning has been widely recognised, but according to our observations, a systematic method to promote higher learning in engineering education does not exist.

In order to have a systematic way to promote higher learning in engineering education despite the economic pressure, the method of cornerstones was implemented. The method is enabled by hierarchical characteristics of natural sciences, since the cornerstones on a certain level of concretisation are only made up from the ones on the lower level of concretisation. The benefits of the method in engineering education are two-fold. First, education is clarified by the identification of cornerstones, since the set of cornerstones includes all the necessary rules for modelling on a certain level of concretisation. Second, the comprehension gets deeper by looking underneath the cornerstones. Then, instead of unchallenged rules, the cornerstones are seen as testable properties. By looking underneath the cornerstones on a certain level of concretisation, students are offered better premises for higher learning and long-term engineering skills. Since this hierarchical structure is inherent for natural sciences, the method of cornerstones can be applied to many fields of engineering. More generally, the substance in engineering education is mostly about identifying the concretisations of modelling.

In this paper, the method of cornerstones was implemented via a simple case study of resistive DC circuits. The example was the simplest one in order to highlight the educational benefits. As can be seen, only slight and precise substance-related modifications are required to increase the role of longterm skills and to create a coherent way to organise the substance in engineering education. Furthermore, a suggestion for coherent and interconnected curriculum structure was also presented. In the end, the driving forces behind this research are the eagerness to promote higher learning, and on the contrary, the worry about excessive emphasis on immediate skills in engineering education. 


\section{References}

Anderson, P. W. (1972). More Is Different. Science, vol. 177, pp. 393-396.

Baez, J. C., Fong, B. (2015). A Compositional Framework for Passive Linear Networks. arXiv:1504.05625v3 [math.CT].

Bok, D. (2006). Our Underachieving Colleges. Princeton University Press.

Brown, G., Bull, J., Pendlebury, M. (2005). Assessing Student Learning in Higher Education. Digital Printing.

Graham, R. (2012). Achieving excellence in engineering education: the ingredients of successful change. The Royal Academy of Engineering.

Jonassen, D., Land, S. (2012). Theoretical Foundations of Learning Environments. Taylor \& Francis.

Keeling, R. P. \& Hersh, R. H. (2011). We Are Losing Our Minds, Rethinking American Higher Education. Keeling \& Associates, LLC.

Kinnari-Korpela, H. (2015). Using Short Video Lectures to Enhance Mathematics Learning-Experiences on Differential and Integral Calculus Course for Engineering Students. Informatics in Education-An International Journal. Vol14_1, pp. 69-83.

Korpela, A., Tarhasaari T., Kettunen L., Mikkonen R., Kinnari-Korpela H. (2015). Towards Deeper Comprehension in Higher Engineering Education: Rethinking "in theory yes, but not in practice". European Journal of Science and Mathematics Education, vol. 3, no. 4, pp. 396-407.

Mortenson, T. G. (2012). State Funding: A Race to the Bottom. American Council for Education, winter 2012.

Porter, W. W., Graham, C. R., Spring, K. A., Welch, K. R. (2014). Blended Learning in Higher Education: Institutional Adoption and Implementation. Computers \& Education, vol. 75, pp. 185-195.

Sheppard, S. D., Macatangay, K., Colby, A. \& Sullivan W. M. (2008). Educating Engineers, Designing for the Future of the Field. Carnegie/Jossey-Bass.

Shulman, L. S. (1972). Those Who Understand: Knowledge Growth in Teaching. Educational Researcher, vol. 12, pp. 4-14.

Sullivan, W. M. \& Rosin, M. S. (2008). A New Agenda for Higher Education, Shaping a Life of the Mind for Practise. Carnegie/JosseyBass.

Wieman, C. E. (2013). Ideas for Improving Science Education. The New York Times, September 2. 